\title{
High-level Abstraction Layers for Development and Deployment of Cloud Services
}

\author{
Binh Minh Nguyen ${ }^{*}$ \\ Institute of Informatics \\ Slovak Academy of Sciences \\ Dúbravská cesta 9, 84507 Bratislava, Slovakia \\ minh.ui@savba.sk
}

\begin{abstract}
Cloud technologies have paved the way for advance of ITbased demand services. It helps decrease operation costs, solve scalability issue and many more user and provider constraints. However, at present, development and deployment of distributed applications on cloud environment becomes a more and more complex tasks. Cloud users must spend a lot of time to prepare, install and configure their applications on clouds. In addition, after development and deployment, the application services almost cannot move from a cloud to others due to the lack of interoperability between them. To address these problems, this paper presents a novel development framework for cloud distributed applications/services. The approach is based on abstraction and object-oriented programming technique, allowing users to easily and rapidly develop and deploy their services into cloud environment. The approach also enables service migration and interoperability among the clouds.
\end{abstract}

\section{Categories and Subject Descriptors}

C.2.4 [Distributed Systems]: Cloud computing-Object oriented architecture, Development framework; D.2.12 [Interoperability ]: Distributed objects

\section{Keywords}

cloud computing, distributed application, abstraction, objectoriented programming, interoperability

\section{Introduction}

The term of cloud computing may not be strange to scientific communities as well industry nowadays, as it grows very fast in the last five years with the support of infrastructures over the network. Cloud computing is described

\footnotetext{
* Recommended by thesis supervisor: Assoc. Prof. Ladislav Hluchý Dr.

To be defended at Institute of Informatics, Slovak Academy of Sciences

(C) Copyright 2013. All rights reserved. Permission to make digital or hard copies of part or all of this work for personal or classroom use is granted without fee provided that copies are not made or distributed for profit or commercial advantage and that copies show this notice on the first page or initial screen of a display along with the full citation. Copyrights for components of this work owned by others than ACM must be honored. Abstracting with credit is permitted. To copy otherwise, to republish, to post on servers, to redistribute to lists, or to use any component of this work in other works requires prior specific permission and/or a fee. Permissions may be requested from STU Press, Vazovova 5, 81107 Bratislava, Slovakia.
}

as a business model for on-demand delivery of computing power, in which consumers pay providers what they used ("pay-as-you-go"). The critical point that distinguishes cloud from other computing paradigms is that cloud itself, to be considered fully virtualized and making illusion of the unlimited resources, enables providers to offer maximal possibility of providing and the users gain utility computing on demand. With the trend towards cloud model, individuals and businesses have changed gradually their habit ways of using the computational resources from their local computers or own servers to data centers of third party.

Conceptually, cloud computing gathers key features like high availability, flexibility and elasticity, that intends to reduce total cost and decrease risk for both users and providers. Today, consumers can buy computation resources, platforms or applications over cloud infrastructures. In the language of this market, the commodities are usually referred to $\mathrm{X}$ as a service (XaaS) paradigm , which mainly includes Infrastructure as a Service (IaaS), Platform as a Service (PaaS) and Software as a Service (SaaS). In principle, cloud-based appliances or services often provide higher availability and lower cost than the traditional IT operations. For this reason, there is now a strong trend of deploying and migrating appliances to cloud. This process could be realized by using PaaS or IaaS. On the one hand, the PaaS clouds provide platform (programming language, databases and messaging) for implementing services and environments for hosting them. The platform also manages the execution of these appliances and optionally offers some advanced features like automatic scaling. Thus, this model allows developers to simply create their cloud services without the need to manually configure and deploy them into virtual machines (VMs). On the other hand, the IaaS clouds provide raw resources (VMs, storages) where the users have full access to the resources and manipulate with them directly in order to create their own platform and deploy services on this.

It can be realized easily that, while PaaS binds developers into certain existing platforms, building cloud services in IaaS will be their choice to meet specific requirements. However, the use of IaaS is perceived as difficult with the developers/users, requiring advance computer skills for the installation and usage. Otherwise, since several commercial cloud systems have been already marketed, the problem of interoperability between these systems also arises, i.e. it would be possible and feasible to move ap- 
pliances from a cloud provider to another, or to deploy an existing appliance on resources provided by several different cloud providers. Such possibility would have very large impact on the competitiveness of providers, as it would require them to offer better quality services at lower prices without forcing customer to rely on their resources through vendor lock-in.

Although several standardizations and solutions in this area have emerged, they have not yet brought any comprehensive solution for the service development and deployment issue on IaaS clouds. Therefore, from the view of general cloud users, they need to have an instrument, which can solve the problem. In this way, cloud service developers will achieve the highest work performance from cloud computing. For example, developers can write, pack and deliver the codes of their services for deployment on various IaaS clouds. In addition, the developers also may manage the services via a unified interface without worrying about the incompatible application programming interfaces (APIs). Thereof, cloud users are not IT experts, who can exploit easily cloud computing to perform their complex works.

The work presented in this paper is dedicated to innovative research and development of an elastic instrument (called high-level Cloud Abstraction Layer - CAL) allowing easy development and deployment of services on resources of multiple infrastructure-as-a-service (IaaS) clouds simultaneously. The CAL provides a total novel approach with emphasis on abstraction, inheritance and code reuse. Then, cloud-based services can be developed easily by extending available abstractions classes provided by the CAL or other developers. The interoperability between different clouds is solved by the basic abstraction classes of the CAL and all services are inherited and benefited from the advantage. The work does not only stop on theoretical work but also continues on applying CAL to build cloud services in order to deal with real problems.

\section{Present State of the Art}

There are economic as well as technological reasons why a service should be developed and deployed on the cloud. On the economic side, reducing costs and business agility are typical business factors for cloud-based appliances. Cloud computing can provide significant cost savings because of the increased utilization resulting from poolÂñing of resources. Otherwise, cloud computing enables rapid delivery of IT services, which increases business efficiency. On the operational side, manageability, performance, and scalability are the typical reasons why service developers consider cloud computing. Besides, cloud computing might offer increased resources, which can lead to performance improvements for the application services. This is especially appealing for appliances with unpredictable or cyclical usage patterns, because a cloud orchestrator can monitor usage and dynamically scale resources up or down.

In addition, service migration is the process of redeploying a service, typically on newer platforms and infrastructures. If the migration is on a compatible platform, the service does not need to be recompiled. In the case of the cloud, services can be migrated from a cloud to others, meanwhile the target infrastructures can be a public, private, or hybrid cloud. The migration of service in cloud environment has many benefits. First, saving the develop- ment cost for programmers, the service can be developed in a private cloud for testing and deployed into a public cloud for using without any obstacles. Second, achieving higher availability rather than the service is deployed on a single cloud. Finally, the migration allows service fault tolerance because it can be deployed on different clouds at the same time.

However, the trend of service development and deployment as well as their migration between clouds is faced with the big challenges:

- PaaS cloud type limits developers/users to concrete platforms and APIs.

- Lack of suitable programming model for service development on IaaS.

- Lack of interoperability between different IaaS clouds.

As mentioned before, using PaaS to develop and deploy cloud services, developers are bound into existing specific platforms and APIs, therefore, building cloud services on IaaS will be their choice to meet complex requirements. The issue here is that the process is perceived as difficult. Concretely, setting-up a service on an IaaS cloud, a developer has to realize step by step as follows:

1. Preparing VM: the developer selects VM image from the repository to get provisioned and launched. Then, he or she configures the features of the VM, including network, firewalls, persistent storage etc.

2. Preparing platform on the VM: if the software packages (e.g. web/app server/runtime) are not installed, the developer needs to install them. This operation involves provision of a database, configuration of data files, log files etc.

3. Deploying service: the developer develops service on his or her created platform.

4. Managing VM and platform: for example, when there is an update or service pack on the operation system (OS), the IaaS provider will not automatically do it for the developer. Instead, this must be done by the developer.

Unfortunately, after development, the services almost cannot move from a cloud to others due to the lack of interoperability between the different clouds. Today, interoperability is another important impact on development of cloud computing. The basic problem of cloud interoperability is that vendor lock-in, in which each cloud provider potentially uses its own stack of technologies consisting for example of hypervisors (e.g. XEN[1], KVM[17] and Microsoft Hyper-V[13]), networking infrastructure, data storage facilities and the corresponding management infrastructure and software support. Cloud users who want to move their appliances/services to other cloud or who want to develop new cloud services on various clouds will be faced with this problem. Hence, a solution or an approach to enable interoperability between clouds becomes more and more practical.

Although some standardization efforts in this area have emerged, such as Open Virtualization Format (OVF)[2], 
Open Cloud Computing Interface (OCCI)[7], and the independent abstraction APIs such as Simple Cloud API[9], Apache Libcloud[10], DeltaCloud[5], jcloud[3] and so on, all of them are still unable to help users create services easily, simultaneously allowing them to deploy among the clouds.

For instance, OVF allows reuse of a VM image standard on diverse clouds. Thus, appliances/services can move among various clouds along with the image. However, creating cloud-based services still require many efforts from developers. As presented above, the process is carried out through numerous complicated steps. This causes timeconsuming and increased cost. Besides, the incompatible APIs issue also brings about the service operation are not guaranteed when they migrate from a cloud to another. Other solution is OCCI that enables cloud users to manage resources from different clouds at the same time. Unfortunately, the users still have to directly connect to VMs in order to create their own platform for service development and deployment. In addition, the weakness of both standardizations above is that they force cloud providers to accept and support their product. Such scenario would have a very large impact on the competitiveness of the cloud providers. Instead of these standards, the abstraction APIs have been created for managing resources from various clouds. The advantage of the abstraction APIs is independent of cloud vendors. However, like OCCI, these abstractions do not help developers develop and deploy services more easily than the traditional way. The developers still have to prepare a platform and develop the services by connecting directly to VMs. Although there are some independent abstraction APIs, which offer support for service deployment via scheduling job mechanism like Simple Cloud API and jcloud, however, in real time, there are drawbacks. Specifically, the life cycle of the applications consists of three stages: submit to computational resources, run or calculate, return results or outputs. After finishing, the used resources will be terminated. Consequently, the API is only suitable for computational applications (e.g. calculation, simulation), not for development and use of cloud services. At present, no APIs have provided the comprehensive solution for both development and deployment tasks.

Clearly, the problem of service development and deployment is a big gap of cloud computing today. It is one of the primary reasons that restrict cloud adoption. So far, there are not any ideas or solutions, which address and resolve this difficult problem.

\section{Methodologies}

\subsection{Abstraction for Cloud Computing}

Theoretically, an abstraction hides details of an entity. In the context of using cloud computing, there are many "entities" that need to be mentioned. However, due to abstraction approach and object-oriented programming (OOP), the entities are encapsulated as data abstractions, which can be used easily via programming methods. Figure 1 shows the hierarchical data abstraction of cloud resources.

The cloud resource abstraction is divided into two parts, namely VM and middlewares. The abstraction can be expressed by tuple $A R$ as follows:

$$
A R=(V M, M)
$$

where $V M$ and $M$ represent virtual machines and middleware abstractions respectively.

Functionally, while the abstraction of VM allows simplifying the use with VM, the abstraction of middlewares enables CAL users (service developers) to manage VM simply under a single interface.

\subsubsection{Abstraction of VM}

From the view of cloud users, an instance (also known as VM) consists of from two components: hardware and software. In CAL framework, they are represented as data in order to be used without understanding details.

$$
V M=\left(H W_{\text {class }}, S W\right)
$$

where $H W_{\text {class }}$ is the hardware class and $S W$ is the software properties.

Like traditional computer, hardware of VM are devices provided as "virtual", consisting of CPU, memory, disk space, OS platform and network. As a rule, IaaS clouds offer their users VM types, in which each type implies a set of the hardware devices. In other words, these types are abstractions of VM hardware. For example, Amazon EC2 enables users to select four types of VM with different attributes[15]. The Small Instance type is equipped with one virtual core CPU, 1.7GB of memory, 160GB of disk storage and 32-bit or 64-bit platform of OS. The other types are equipped with more powerful hardware and bring higher performance for VM. Besides, most of public clouds provide the network by associating automatically a public IP address with VM. For open source clouds, there are some exceptions

In the heterogeneous environment of multiple clouds, the existing VM types provided by different infrastructures can have small differences. Therefore, to use these clouds simultaneously, CAL defines a high level abstraction of VM hardware by classifying similar VM types into common classes. The VM types from different cloud infrastructures are classified into general hardware classes in abstraction level by the function:

$$
H W_{\text {class }}=\left\{i \in N, H W: f\left(H W_{i}\right)\right\}
$$

where $f$ is the classification function of VM types.

Software of VM is the collection of programs that provides instructions for controlling the machine what to do and how to do it. In the context of virtualization, the software refers to OS and applications. In the same way of hardware abstraction, CAL also abstracts VM software in form of data strings.

$$
S W=\left(B_{a s e S W} A p p_{1}, A p p_{2}, A p p_{3}, \ldots, A p p_{i}\right)
$$




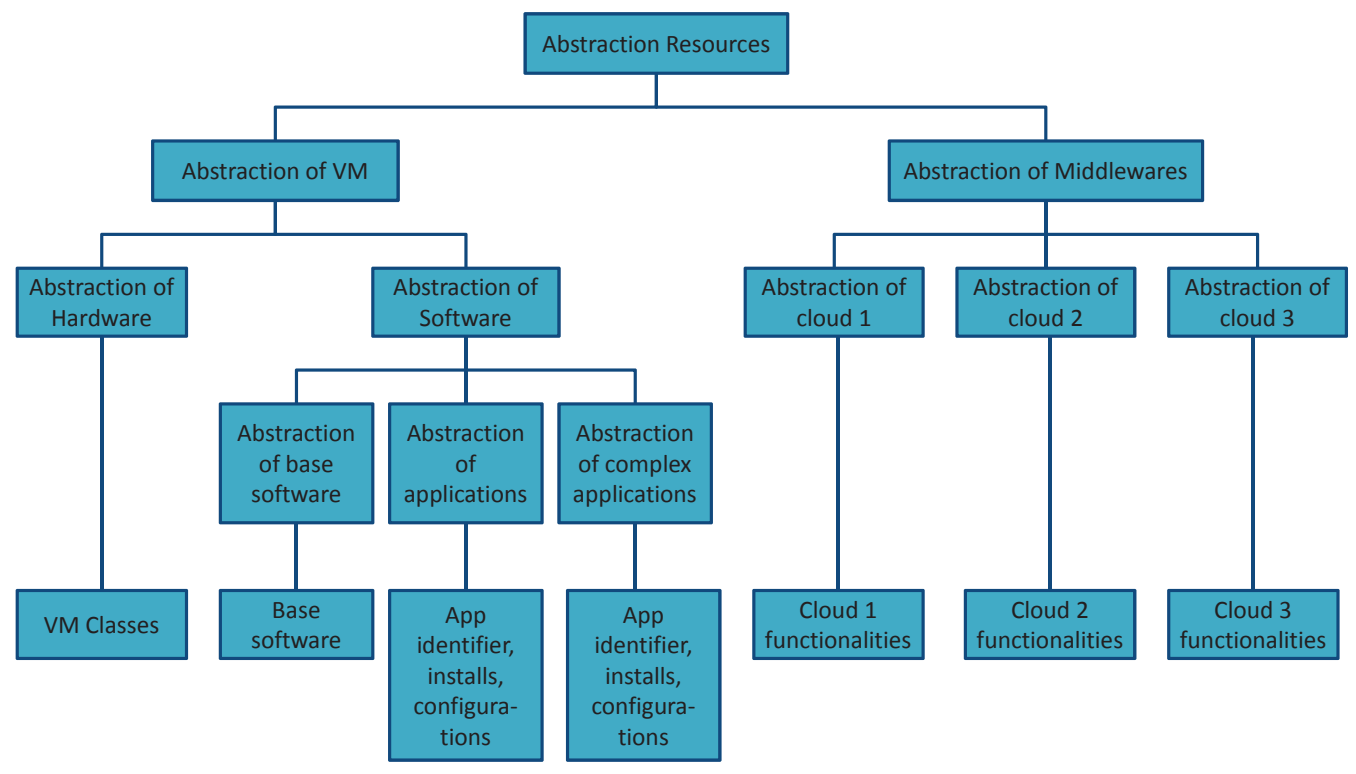

Figure 1: High-level abstraction of cloud resources

where $B a s e S W$ is abstraction of base software and $A p p_{i}$ is abstractions of applications.

The base software content includes an operation system and a set of standard software packages for the operating system. The base software must provide functionalities for data transfer from/to VM and remote execution of commands with administrative privileges on the VM. On the other hand, the abstraction of an application in cloud environment can be expressed by a tuple $A p p$ as follows:

$$
A p p=\left(a_{\text {identifier }}, a_{p} p_{\text {install }}, a_{\text {config }}\right)
$$

where $a_{p p} p_{\text {identifier }}$ is identification of application, app install indicates installation package of the application and $a p p_{\text {config }}$ is initial setting for the applications.

There is some information, which is required to abstract the applications covering their identifiers, installation package locations and configurations. The application configurations are initial settings for an application. Application configuring allows users to choose suitable parameters, ensuring the application work as their desire. Besides application configurations, in most cases, applications can be installed with default configurations via advance packaging or manager tools (e.g. apt, git in Ubuntu/Debian) and they can be downloaded from software libraries. MySQL, TomCat, Apache, Java and Python libraries, etc. are typical examples of applications. However, some applications require indicating their paths or locations during installation process (e.g. user applications).

In the level of abstraction, an application is identified by abstract data string that can be a word or term. The abstract data thus discriminates applications with each others. Additionally, the application abstraction normally are expressed together with its development versions, which are used to assign in increasing order and correspond to new release in the application.
Otherwise, there are also complex applications that are constituted from many application components. In general, the complex applications comprise a core and additional supplements. The process of installing these applications is carried out one after another as follows: installation of the core, installation of the supplements and configuration of whole applications. For example, to build a web server (a complex application), developers have to install and configure Apache, MySQL and PHP (single applications) in turn. Then they configure parameters of the whole server such as privacy, web directory, etc..

In CAL framework, the VM will be created with the base software, so the applications will be installed/configured on the base software in the order they are defined in the abstraction. The installation package and configuration of the applications will be transferred to the VM via data transfer functionality of base software. Similarly, the installation commands of the software will be executed on $\mathrm{VM}$ via remote execution of base software.

\subsubsection{Abstraction of Middlewares}

One of the objectives of CAL is to manage resources from many cloud middlewares at the same time. Currently, most of the clouds provide APIs, which are efficient management means for users. Many cloud tools have been built based on the APIs. Essentially, API is an abstraction of cloud functionalities such as creation, termination, VM snapshot and so on. As mentioned before, API has many different forms and each cloud usually offers a separate API that differs from others. To achieve the objective, CAL implements the APIs as data abstractions. In this way, each cloud has an abstraction for its own and the abstraction provides functionalities to manage resources of the cloud. At the higher level, abstraction of middlewares encapsulates the API data abstractions in the form of new type, allowing manageability of multiple middlewares under a single unified interface. The abstraction of middlewares $\mathrm{M}$ is set of common cloud functionalities provided by different infrastructures. 


$$
M=\left\{k_{1}, k_{2}, k_{3}, \ldots, k_{n}\right\}
$$

where $k_{i}$ are cloud functionalities.

Abstraction of middlewares can be considered as a common denominator of functionalities provided by various clouds. Basically, prevalent cloud functionalities include: creation, termination, VM description, snapshot creation and restoration. They are basic management functions for developing cloud service on VM. Most of them are supported by every cloud through their APIs. Therefore, the functionalities of middleware abstraction are shaped from separate API implementation for each cloud.

Besides the basic functions, there are other functionalities such as IP allocation and association, user key creation, deletion, etc. According to requirements of CAL users, the implementation of API data abstractions can be extended in order to add more functions for the abstraction of middlewares.

\subsection{OOP for Abstraction in Cloud Environment}

The signification of encapsulationas well as polymorphism in OOP context is similar to the meaning of the abstraction approach. It shows the feasibility in applying OOP to create abstractions for cloud computing in order to hide implementation details of different clouds under a single unified interface.

Specifically, using the encapsulation characteristic of OOP, cloud resource abstraction $A R$ can be represented as a class due to programming languages (e.g. Python, Java). The class provides management functions for CAL users via its interface and the users do not need to care about implementation details of abstraction components ( $V M$, $M)$. The $V M$ abstraction enables CAL users to create easily VMs via unified parameters $\left(H W_{\text {class }}\right.$ and $\left.S W\right)$ in OOP methods even though the VMs belong to different cloud infrastructures. In the similar way of using encapsulation for $A R$ and $V M$ above, the rest of abstractions such as $H W_{\text {class }}, S W$, Base $S W, A p p$ and $M$ also can be designed and used simply through OOP.

On the other hand, due to the polymorphism characteristic of OOP, CAL functions can be used in different cases. This helps avoid programming functions that have identical features. The polymorphism also enables CAL users (cloud service developers) to develop new functionalities for their service based on combining existing CAL functions without programming again.

According to inheritance feature, using OOP for implementing abstraction approach allows creating child abstraction layers based on an initial abstraction layer. Thus, the child layers inherit functionalities of the initial layer without having to rewrite the code. More importantly, a child layer can become the parent layer of their child layers. It means that the expansions of abstraction layers are unlimited, depending on the requirement of users, in which one layer of abstraction looks at an object-oriented application as consisting of service providers and serviceconsumers or clients. Clients of a server are interested only in what the server provides (its behavior) and not how it provides it (its implementation). The client only needs to know the public interface of a class it wants to

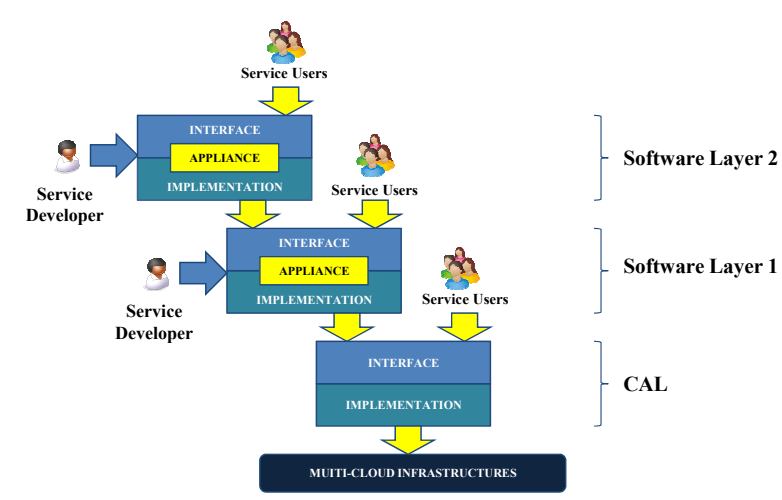

Figure 2: Software layering of CAL development framework

use - what methods it can call, their input parameters, what they return and what they accomplish.

Based on the inheritance characteristic of OOP, one software layer, which was created by a developer, can also be used and further extended by other developers. Figure 2 shows the inheritances by multiple service developers relying on CAL. In this figure, the first developer defines software layer 1 with new functionalities on demand from his or her users. In other words, software layer 1 inherits all functions and hides implementation details of CAL in its functionalities. Similarly, the second developer defines functionalities for software layer 2 over the layer 1 by inheriting and hiding layer 1 functionalities. As the result, each software layer is practically a platform-as-a-service by itself, because users only use the services via a clear interface provided by the developers without interacting directly with VMs on clouds. For concrete example, a developer creates a LAMP stack (Linux-Apache-PHP-MySQL) layer that is equivalent to web-hosting platforms for his or her service users. Another developer can use this LAMP layer to provide web applications (e.g. wiki, forum) without manipulating with the cloud infrastructures.

Since higher software layers are independent from cloud infrastructures, if CAL correctly operates with multi-clouds, any services using CAL will also operate correctly on the infrastructures. Generalized interoperability problem of cloud systems can be reduced into the interoperability problem of selected software layers in this development framework. Developers can easily move services (software layers) among various clouds without depending on providers.

\section{Designing CAL}

Architecture of CAL is depicted in Figure 3. It contains three components: interface, drivers and data repository. The interface provides interaction between CAL and its users. Therefore, from the view of service developers, it is visible. Otherwise, the drivers and data repository are designed to hide under the interface. Since the number of variations of VMs is often limited (some major OS flavors or VM types), making these functionalities operate correctly on a given cloud infrastructure can be solved with reasonable efforts.

\subsection{CAL Interface}

The interface is designed to provide functional abstractions of the cloud resources. Using the CAL, service de- 


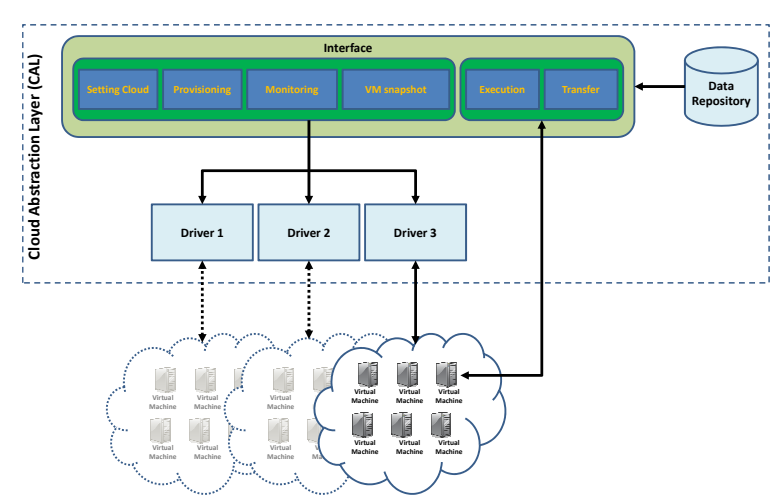

Figure 3: CAL architecture

velopers just reuse the functions of CAL interface to create their services. For easier understanding, the functions are divided into numerous groups, consisting of:

- Setting Cloud: enables developers to set which cloud will be used. This group has only setCloud() function.

- Provisioning: consists of start() and stop() function to create and terminate the VMs.

- Monitoring: getting actual information of the machines (cloud provider, IP address, ID instance and so on) by status() function.

- Execution: running commands on VMs by execute() function.

- Transfer includes two functions: put_data () to upload and get_data() to download data from the VMs.

- VM Snaphost: creates/restores snapshot of VM into an image. The group involves create_snapshot() and restore_snaphsot() function.

While the functions of Provisioning, Monitoring and VM Snapshot group use cloud APIs to implement actions of VM, Execution and Transfer do not. The reason is that there are no APIs that support the operations. The advantage of CAL is to provide developers with functionalities in order to overcome the restrictions. In this way, CAL abstracts the connection, realization process and hides implementation details by the functions of Execution and Transfer. As the result, developers can run commands or upload, download their data without having to directly connect to VM. Since VMs are normally connected via public IP addresses under authentications (e.g. key pair, usernames, passwords), Execution and Transfer functionality are used for all VMs even when they belong to different clouds.

\subsection{CAL Drivers}

CAL may have many drivers. Each driver is a module of API data abstraction, which allows CAL to manage resources from a cloud via its API. As mentioned before, the number of drivers is in proportion to the number of clouds that CAL supports. For example, if CAL supports Amazon EC2 and OpenNebula[8] cloud, it means

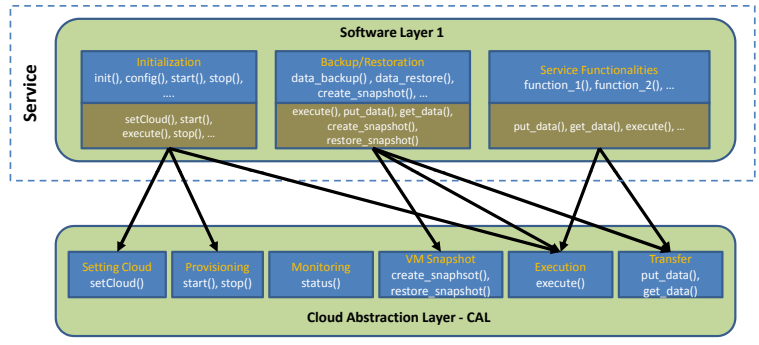

Figure 4: Inheritance feature of CAL

that CAL will have two drivers. When CAL needs to expand its support for other clouds, the only thing to do is creating the new driver without changing the original codes, including interface and existing drivers. The relationship between each driver and the interface within CAL is similar to the relationship of a hardware device (e.g. graphic card) and an application or OS in traditional computer, where hardware driver acts as a translator between the device and application. Indeed, the drivers of CAL also act as intermediaries to translate the developersâĂŹ commands (via functionalities of CAL interface) to clouds. Because of the incompatibility of APIs, every cloud must have a driver of its own.

Because CAL provides only basic functionalities of VM, the drivers thus do not need to implement all API actions. The CAL functionalities are implemented through APIs covering Provisioning, Monitoring and VM snapshot.

\subsection{CAL Data Repository}

Besides interface and drivers, data repository plays the part of memorizing parameters related to $\mathrm{VM}$ and services during CAL usage. For example, the repository stores name of default baseSWbaseSW, VM snapshot, VM IP address, VM ID and other configuration parameters (e.g. administrator name, password, database name). A data repository for CAL brings many advantages for service developers, including:

- Optimization of the CAL usage - the developers do not have to repeat the values of VM IP address, VM ID or other parameters (e.g. service password) in every functional abstraction. All of them are stored automatically in the data repository, thus simplifying CAL use.

- Elimination of repeating service installations, since VM snapshot names are stored in the repository. Service developers can optimize the VM start process by checking existing services. If a service is available, its snapshot will be used to create VM. Otherwise, the developers will deploy developed services in the normal way (with a base $S W$ ).

\subsection{Inheritance of CAL Functions}

As mentioned before, developers can easily create cloud services by using CAL. This is shown in Figure 4. The developers just have to inherit the existing functional abstractions of CAL for creating new service functions, which can be grouped as follows:

- Initialization: Developers just reuse the Setting Cloud functionality of CAL to select cloud in or- 
der to deploy their services. Then they create a $\mathrm{VM}$ on the cloud by using the functions of Provisioning. The developers can add OS commands to install software packages on newly created VM by Execution. Otherwise, they also can upload their initial data or applications into the VM by Transfer.

- Backup/Restoration: comprise service functions to perform two tasks:

- Creating/restoring VM snapshot for the service. For this purpose, the developers inherit VM Snapshot functionalities.

- Creating/restoring user backup data, the developers reuse Execution and Transfer.

- Service functionalities: Developers can create other functions for their services by reusing and combining the existing functional abstractions of CAL. For example, for database servers, they can add a number of functions to import database, make query, and so on. The database functions are programmed based on Execution and Transfer.

One of the most important things is that during development, the developers do not need to use any specificmiddleware APIs or connect directly to VMs as well. They only inherit the functions provided by CAL. The developers can thus simply select the target cloud to deploy their service without having to worry about incompatible cloud systems. Meanwhile, their user (distinguish from the developer) will just use the service via Initialization, Backup/Restoration and service functionalities. The user would not require caring about how and where the service is developed and deployed.

\section{Case Studies}

\subsection{Experimental Setup}

Our current implementation of CAL prototype bases on the installations of three middlewares: OpenStack[12] Folsom release, Eucalyptus 2.0.3 (open source version) [11] and OpenNebula 3.8. The purpose of this setup is to provision VMs that belong to those middlewares at the same time. Tests are successful if and only if all VMs have SSH access. For that purpose, all three middlewares are installed and configured separately in our servers. Each of them consists of a controller node, a management network (switch) and at least two compute nodes. For controller nodes, each server blade is equipped with processor Xeon including 16 cores $(2.93 \mathrm{GHz}), 24 \mathrm{~GB}$ of RAM and 1TB hard drive, meanwhile for compute nodes, each server blade is equipped with processor Xeon with 24 cores $(2.93 \mathrm{GHz}), 48 \mathrm{~GB}$ of RAM and $2 \mathrm{~TB}$ hard drive. Linux is installed for all servers as OS. KVM hypervisor is used for all three systems. An Ubuntu 12.04 images are created and deployed on the clouds. While OpenStack, Eucalyptus are configured with Glance [14] and Walrus [4] respectively as internal image storage services, OpenNebula uses non-shared file systems [16] with transferring image via $\mathrm{SSH}$ for test purpose.

\subsection{Development and Deployment of Cloud Monitor- ing Service}

The realistic services are the best way to show and demonstrate the CAL effects. In the direction, this section presents the development and deployment of a cloud monitoring service for distributed systems based on CAL. Additionally, the layering feature of the services is also demonstrated here.

A monitoring service must have the ability to provide data usage of components within VM and fundamental aspects of appliances in that VM. As a consequence, the service needs to be adaptable and extensible in order to support the expanding functionality. To address all of the requirements and functionalities, the main features for service to be taken into account are as follows:

- Scalability: ensures that the monitoring can cope with large numbers of VMs.

- Adaptability and extensibility: ensure the monitoring framework can adapt to varying computational load.

- Federation: ensures that any VM which resides on various clouds can be monitored.

To establish such features, the monitoring service is built based on Nagios core framework application. First reason of the choice is that Nagios core is a powerful monitoring solution that is used by large IT organizations [6]. Otherwise, it also has been one of the most prevalent monitoring solutions known in open source community. The second reason is that the core does not contain any checking tools (called Nagios add-ons) at all. Due to this important feature, Nagios core can provide a robust, reliable and extensible framework for any type of check that a user can come up with. Currently, the most general Nagios add-ons are:

- NRPE (Nagios Remote Plugin Executor) provides the ability to monitor VM metrics (e.g. disk space, CPU workload).

- Nagios BPI (Bussiness Process Inteligence) creates a way to visualize business process health by grouping hosts and services together, and creating rules to discern the true health of the network infrastructure as it relates to the business.

- Nagiosgraph extracts information from the Nagios output, processes it, and then insert it into one or more round-robin database (RRD) files. The plugin also embedded RRD file directly into Nagios interface in form of graphs as trend reports.

\subsubsection{Nagios Monitoring Framework Service}

Using Python language the high-level abstraction layer is represented as "CAL" class, which provides the basic functions of VM. For each cloud infrastructure, we respectively define separate classes: Openstack, Eucalyptus and OpenNebula, which are the drivers of these clouds.

Figure 6 describes the software layering of monitoring framework service. In the context of the implementation using CAL, the framework can be considered as platformas-a-service layer that can be used for many different monitoring purposes. Based on the layer, developers can program to provide various specific monitoring services by installing Nagios add-ons. In this way, the Nagios add-on 


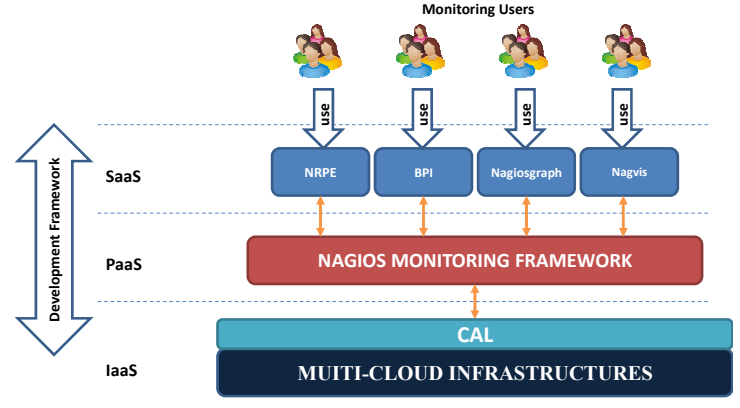

Figure 5: Nagios monitoring framework service

services are equivalent as software-as-a-service layer over the monitoring framework.

Although Nagios core is a large and complicated application that normally requires expert computer skills to install and configure, using CAL, the development process of monitoring framework service can be carried out quite easily. The monitoring framework service is implemented as Nagios class. Service methods are created by reusing (inheritance mechanism) the CAL methods.

The monitoring framework service is implemented to operate on Linux server. Installation commands of Nagios are predefined in configuration files, which are uploaded to VM.

\subsubsection{NRPE Monitoring Service}

As mentioned before, NRPE is one of the most popular add-ons of Nagios core. This section describes the implementation and use of NRPE service, which can monitor resources and services running on cloud VMs. In the development framework, NRPE monitoring service is equivalent to SaaS layer because it is developed based on a platform (Nagios monitoring framework service). NRPE add-on provides a lot of different monitoring functionalities such as check disk, CPU load, memory, users, number of running/zombie process, service status (http, apache, MySQL), and so on. All of the functions are defined in the object definition files. In this way, users have the ability to choose which resource or services are monitored.

The NRPE functions are implemented as methods of NRPE class. Similar to monitoring framework service, the installation commands also are predefined in configuration files that are uploaded to VM to execute. Otherwise, to remove or stop NRPE service on monitoring framework, the removing file with un-installation commands defined inside is used.

Transparently, during the service development, only monitoring framework service functions are used. Developers do not need to know about VM, SSH, SCP commands as well as implementation details of the monitoring framework service. The NRPE monitoring is called through the following commands:

\subsection{Experimental Results}

To evaluate operation of CAL, Nagios framework monitoring service and NRPE service, the deployment process is tested on three cloud installations with various VM types. The experimental measurement is repeated 20 times for each of the VM type of each cloud. The

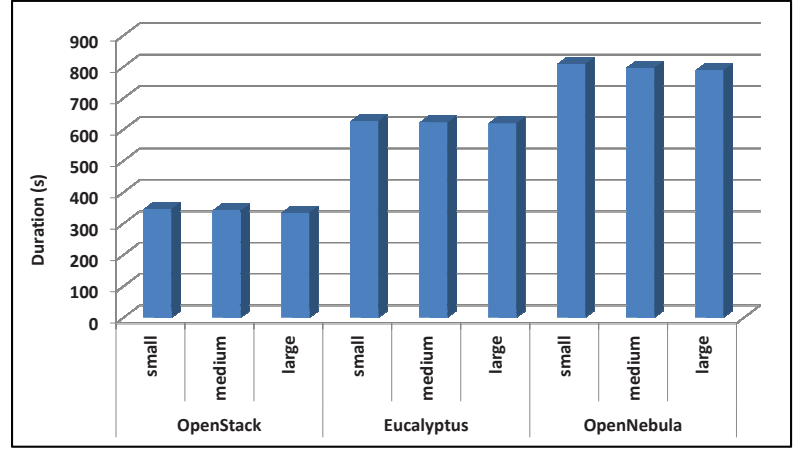

Figure 6: Deployment time of Nagios framework monitoring service

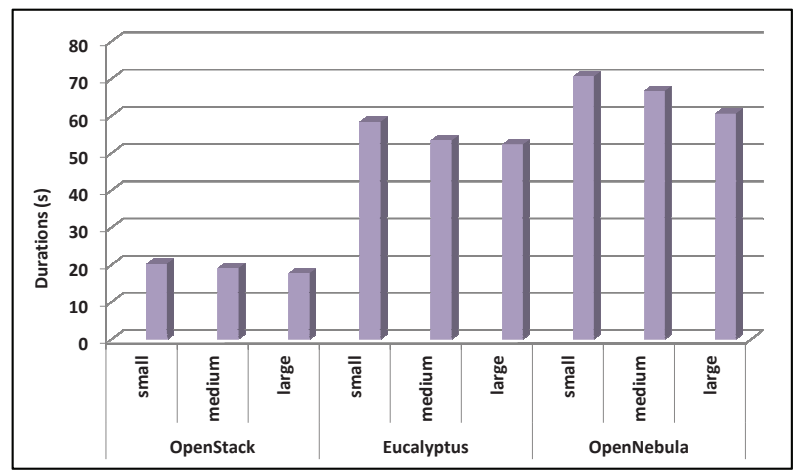

Figure 7: Deployment time of NRPE monitoring service

NRPE service deployment process is experimented based on Nagios framework layer, which already has been deployed into the VMs of three cloud middlewares. The average times are summarized in Table 1. The duration time is calculated in seconds.

Figure 6 represents the deployment time results of Nagios framework monitoring service described in Table 1 . There are some observations that can be made from inspecting the results: first, Nagios framework monitoring service can operate well on all three cloud infrastructures at the same time without changing its implementation codes. Second, in comparison between OpenStack with the rest, the middleware gains better performance. This is demonstrated by the experiment results presented in table above. Specifically, the deployment process of $\mathrm{Na}-$ gios core framework service using OpenStack is faster than using Eucalyptus (approx. 49\%) and using OpenNebula (approx. 57\%).

The deployment time results of NRPE service are illustrated by diagram in Figure 7. The experiment proves that NRPE service operates well on Nagios monitoring framework deployed on the different clouds. More importantly, the development and deployment process of NRPE service emphasize that CAL software layering feature as well as the feasibility of our approach.

In summary, the case studies give the following important outcomes:

- CAL operates well with the installed cloud middlewares. It provides the development and deployment 


\begin{tabular}{|c|c|c|c|}
\hline & VM Type & $\begin{array}{c}\text { Nagios framework } \\
\text { monitoring service }\end{array}$ & NRPE service \\
\hline \multirow{3}{*}{ OpenStack } & small & 346.296 & 20.276 \\
\cline { 2 - 4 } & medium & 341.407 & 19.128 \\
\cline { 2 - 4 } & large & 334.121 & 17.765 \\
\hline \multirow{3}{*}{ Eucalyptus } & small & 626.204 & 58.421 \\
\cline { 2 - 4 } & medium & 621.812 & 53.357 \\
\cline { 2 - 4 } & large & 618.698 & 52.245 \\
\hline \multirow{3}{*}{ OpenNebula } & small & 808.742 & 70.603 \\
\cline { 2 - 4 } & medium & 796.186 & 66.679 \\
\cline { 2 - 4 } & large & 788.233 & 60.603 \\
\hline
\end{tabular}

Table 1: Deployment time results of Nagios framework monitoring service

framework for service developers and users.

- The framework enables software layering, in which each layer is the foundation for new layers over it. During the development, deployment and use process, they only inherit and reuse available functions of preceding layers instead of directly using any functionality of VM and cloud middlewares.

- The developed services were tested and work well.

- The developed services are independent from cloud infrastructures and they have the ability to be deployed on different clouds at the same time.

- The process of deploying services takes a long time, however, since the process is realized automatically, the time for deployment is always less than manipulation of the traditional approaches.

Beside the case studies with Nagios monitoring service and its add-ons, we built a number of other cloud services, namely cloud database and web-hosting services. Similar to the studies presented in this papers, the other services developed in CAL framework also can be operated well with our cloud installation middlewares. This demonstrated that our approach has the ability to apply widely in developing and deploying interoperable services in cloud environment. Relying on that CAL solves one of the outstanding problems for cloud computing today.

\section{Conclusions}

The study described in this paper was carried out in the emerging context of cloud computing with issue linked to the development and deployment of services over IaaS clouds at once. In this direction, this research focused on building a tool that enables every service to be able to develop and deploy into different clouds without requiring additional complicated work form developers and special supports from providers or middlewares. The tool was named "CAL" that brings the following results:

1. Mechanism to simply develop and deploy services in different IaaS clouds: cloud services are treated as objects with strongly defined interfaces. The services thus are developed based on abstraction approach and OOP. CAL acts as development framework for the services. It includes basic functionalities of VMs that belong to many clouds. With the available functions, developers easily define the interface for their services without the necessity to access application code directly; in other words, without connecting directly to VMs to configure, install the applications. In addition, developers also can customize, extend services developed by others to create new services without access to original codes. Due to CAL, the services are developed and used with minimum VM manipulations. The mechanism thus allows simplification in development and deployment of services within cloud environments.

2. Mechanism to enable interoperability of cloud computing services: service codes that are implemented using CAL can be packed and delivered for deployment on various clouds without obstacles such as incompatible APIs, virtualization technologies or other middleware components. This means, the codes are written once but can be used on many clouds without re-implementation. In this way, this mechanism allows achieving the interoperability, which is one of the invaluable features for cloud computing today.

3. Adding values for cloud providers: due to the abstraction approach of CAL, cloud services are developed and deployed independently of providers. So, the providers do not have to change in order to support the services. This still ensures the competitiveness among the vendors. Otherwise, they achieve benefits when service developers/users exploit cloud resources more easily, exciting the growth of consumer market for IaaS cloud providers.

Since CAL services are independent from underlying infrastructures, they can be published in a marketplace that allows other service developers or pure users to download and use them without coding. Consequently, in the near future, we will continue to build the marketplace for CAL services.

Acknowledgements. This work is supported by projects SMART II ITMS: 26240120029, VEGA No. 2/0054/12, CRISIS ITMS: 26240220060, CLAN No. APVV 0809-11.

The author would like to thank his supervisor Assoc. Prof. Ladislav Hluchý Dr. for his valuable advice and comments.

\section{References}

[1] P. Barham, B. Dragovic, K. Fraser, S. Hand, T. L. Harris, A. Ho, R. Neugebauer, I. Pratt, and A. Warfield. Xen and the art of virtualization. ACM SIGOPS Operating Systems Review, 37(5):164-177, 2003. 
[2] S. Crosby, R. Doyle, M. Gering, M. Gionfriddo, S. Grarup, S. Hand, and D. Hiltgen. Open virtualization format specification. vol. DSP0243(1(0)), 2010.

[3] D. Franz, J. Tao, H. Marten, and A. Streit. A workflow engine for computing clouds. In The Second International Conference on Cloud Computing, GRIDs and Virtualization proceedings, pages 1-6, 2011.

[4] A. M. Lonea, D. E. Popescu, and O. Prostean. A survey of management interfaces for eucalyptus cloud. In 7th IEEE International Symposium on Applied Computational Intelligence and Informatics (SACI) proceedings, pages 261-266, 2012.

[5] J. L. Lucas-Simarro, R. Moreno-Vozmediano, R. Montero, and I. Llorente. Scheduling strategies for optimal service deployment across multiple clouds. Future Generation Computer Systems, 2012

[6] M. Ma, D. Kouril, M. Prochazka, C. L'Orphelin, O. Lequeux, P. Veyre, C. Triantafyllidis, C. Kanellopoulos, and P. Korosoglou. Egi security monitoring. In International Symposium on Grids and Clouds proceedings, volume 1, 2012.

[7] T. Metsch and A. Edmonds. Open cloud computing interface infrastructure. Standards Track, no. GFD-R in The Open Grid Forum Document Series, Open Cloud Computing Interface (OCCI) Working Group, Muncie (IN), 2010.

[8] D. Milojicic, I. Llorente, and R. Montero. Opennebula: A cloud management tool. Internet Computing, 15(2):11-14, 2011.

[9] D. Molnar and S. Schechter. Self hosting vs. cloud hosting: Accounting for the security impact of hosting in the cloud. In 9th Workshop on the Economics of Information Security (WEIS) proceedings, 2010.

[10] S. Mu, K. Chen, P. Gao, F. Ye, Y. Wu, and W. Zheng. ulibcloud: Providing high available and uniform accessing to multiple cloud storages. In ACM/IEEE 13th International Conference on Grid Computing proceedings, pages 201-208, 2012.

[11] D. Nurmi, R. Wolski, C. Grzegorczyk, G. Obertelli, S. Soman, L. Youseff, and D. Zagorodnov. The eucalyptus open-source cloud-computing system. In 9th IEEE/ACM International Symposium on Cluster Computing and the Grid proceedings, pages 124-131, 2009

[12] TaheriMonfared, Aryan, and M. G. Jaatun. As strong as the weakest link: Handling compromised components in openstack. In 3rd International Conference on Cloud Computing Technology and Science proceedings, pages 189-196, 2011.

[13] A. Velte and T. Velte. Microsoft Virtualization with Hyper-V. McGraw-Hill, New York, 2009.

[14] G. von Laszewski, J. Diaz, F. Wang, and G. C. Fox. Comparison of multiple cloud frameworks. In IEEE Cloud 2012 proceedings, pages 734-741, 2012.

[15] G. Wang and T. E. Ng. The impact of virtualization on network performance of amazon ec 2 data center. In International Conference on Computer Communications proceedings, pages 1-9, 2010.

[16] X. Wen, G. Gu, Q. Li, Y. Gao, and X. Zhang. Comparison of open-source cloud management platforms: Openstack and opennebula. In 9th IEEE International Conference on Fuzzy Systems and Knowledge Discovery proceedings, pages 2457-2461, 2012.

[17] B. Zhang, X. Wang, R. Lai, L. Yang, Z. Wang, Y. Luo, and X. Li. Evaluating and optimizing i/o virtualization in kernel-based virtual machine (kvm). In Network and Parallel Computing Proceedings, pages 220-231. Springer Berlin Heidelberg, 2010.

\section{Selected Papers by the Author}

Binh Minh Nguyen, Viet Tran and Ladislav Hluchy. A Generic Development and Deployment Framework for Cloud Computing and Distributed Applications. Computing and Informatics, vol. 32, no. 2, 2013.

Binh Minh Nguyen, Viet Tran and Ladislav Hluchy. Abstraction Layer for Development and Deployment of Cloud Services. Computer Science, vol. 3, no. 3, p. 80-88, 2012.

Binh Minh Nguyen, Viet Tran and Ladislav Hluchy. Developing and Deploying Cloud Services Based on Abstraction Approach.
Digital Information Management, vol. 10, no. 3, p. 192-199, 2012.

Binh Minh Nguyen, Viet Tran and Ladislav Hluchy. Abstraction Layer for Cloud Computing. Scalable Computing: Practice and Experience, vol. 12, no. 3, p. 371-374, 2011.

Binh Minh Nguyen, Viet Tran and Ladislav Hluchy. High-level Abstraction Layers for Development and Deployment of Cloud Services. In 4th International Conference on Networked Digital Technologies - NDT 2012, CCIS 293, pages 208-219, Dubai, UAE, 2012. Springer.

Binh Minh Nguyen, Viet Tran and Ladislav Hluchy. Development Framework for Cloud Services. In International Conference on Network Computing and Information Security - NCIS 2012, CCIS 345, pages 137-147, Shanghai, China, 2012. Springer.

Binh Minh Nguyen, Viet Tran and Ladislav Hluchy. An Abstraction Model for High-level Application Programming on the Cloud. In 1st International Conference on Computer Science Applied Mathematics and Applications - ICCSAMA 2013, SCI 479, pages 295-306, Warsaw, Poland, 2013. Springer.

Binh Minh Nguyen, Viet Tran and Ladislav Hluchy. A Novel Approach for Developing Interoperable Services in Cloud Environment. In 27th of the International Conference on Information Networking - ICOIN 2013, pages 232-237, Bangkok, Thailand, 2013. IEEE Computer Society.

Binh Minh Nguyen, Viet Tran and Ladislav Hluchy. A High-level Abstraction Layer for Cloud Computing. In 6th International Conference on Computer Sciences and Convergence Information Technology - ICCIT 2013, pages 446-449, Jeju, South Korea, 2011. IEEE Computer Society.

Binh Minh Nguyen, Viet Tran and Ladislav Hluchy. Abstraction Approach for Developing and Delivering Cloud-based Services. In International Conference on Computer Systems and Industrial Informatics, - ICCSII 2012, 6 pages, Dubai, UAE, 2011. IEEE Computer Society. 